\title{
РАСПОЗНАВАНИЕ ИЗОБРАЖЕНИЙ ЭЛЕМЕНТОВ ЗЕРНОВЫХ СМЕСЕЙ МЕТОДАМИ ГЛУБОКОГО ОБУЧЕНИЯ С ИСПОЛЬЗОВАНИЕМ БИБЛИОТЕК КЕRAS И TENSORFLOW
}

\author{
А. А. Крыловецкий, Д. М. Суходолов \\ Воронежский государственный университет
}

Поступила в редакцию 21.06.2018 г.

\begin{abstract}
Аннотация. Методом вычислительного эксперимента исследована возможность использования нейросетей глубокого обучения для распознавания изображений элементов зерновых смесей. Проведено сравнение результатов для трех различных архитектур, реализованных в пакетах VGG16, VGG19 и MobileNet на наборе изображений зёрен различных растений. Выявлен относительный вклад морфологических, цветовых и текстурных признаков в решение задачи классификации изображений зерен с помощью нейросетей глубокого обучения.

Ключевые слова: обработка изображений, анализ изображений, глубокое обучение, машинное обучение, нейросеть, классификация зерновых смесей.

Annotation. The possibility of using deep learning neural networks for recognizing images of elements of grain mixtures was investigated using the method of computational experiment. Comparison of results for three different architectures - VGG16, VGG19 and MobileNet on a set of images of grains of various plants was made. The relative contribution of morphological, color and textural features to the solution of the problem of classification of grain images with the help of deep learning neural networks is revealed.

Keywords: image processing, image analysis, deep learning, machine learning, neural network, classification of grain mixtures.
\end{abstract}

\section{ВВЕДЕНИЕ}

В настоящее время системы машинного зрения нашли широкое промышленное применение. Компьютерное зрение используется в разных областях производства для управления различными процессами, в научных исследованиях для создания новых технологий, таких как виртуальная реальность. В зерновой промышленности технологии машинного зрения стали достаточно интенсивно применяться в начале XXI века. Использование методов обработки изображений в агропромышленном комплексе связано с определенными проблемами. В частности, главным препятствием являются значительные вариации размера, формы, цвета и текстуры биологических объектов. Впервые в 1980 году была создана эффективная система анализа изобра-

(с) Крыловецкий А. А., Суходолов Д. М., 2018 жений в агро-пищевой промышленности для выполнения простых задач, таких как проверки и сортировки [1]. На начальном этапе в подобных решениях использовались монохромные датчики изображения низкого разрешения $(128 \times 128$ пикселей $)$ и простые методы обработки изображений [2]. В начале 90-х годов прошлого века в аграрной и продовольственной промышленности начали применяться системы обработки цветных изображений благодаря появлению в индустрии твердотельных датчиков изображений и недорогих высокоскоростных микрокомпьютеров.

Основы для разработки эффективных систем машинного зрения для сельского хозяйства и производства продуктов питания были заложены в целом ряде работ Barker D. А. с соавторами [3-7], D. R. Draper с соавторами [8-10], в работе Lai F. S. [11], в работах D. S. Jayas и S. Majumdar с соавторами [12-19], в ряде работ D. G. Myers, M. Neuman, 


\section{А. А. Крыловеикий, Д. М. Суходолов}

J. Paliwal, H. D. Sapirstein, S. J. Symons и соавторов [20-26], в работах I. Zayas с соавторами [27-30]. В этих исследованиях были разработаны основные принципы использования методов анализа изображений и распознавания образов в такого рода задачах. Например, в работе [31] применялись методы машинного зрения для обнаружения и классификации поврежденных грибковой инфекцией соевых бобов. Соотношение интенсивностей красного и синего, красного и зеленого, зеленого и синего были взяты в качестве входных признаков. Было достигнуто от 77 \% до $91 \%$ точности при классификации зараженных образцов.

Основные методы обработки изображений, используемые в системах машинного зрения в настоящее время для оценки качества сельскохозяйственной продукции, были описаны в работах [16-19, 32-34]. В частности, предложены алгоритмы извлечения информации о размере, форме, цвете, вычисления дескрипторов формы и цвета, дескрипторов Фурье и текстурных дескрипторов, основанных на матрицах сочетаемости яркостей (GLCM) и длин серий (GLRM). Были разработаны программные реализации для извлечения 230 признаков из цветных изображений зерновых объектов.

Большинство систем машинного зрения используют для решения задач идентификации и классификации небольшое количество дескрипторов. В ряде работ предлагается использовать различные наборы дескрипторов и разные классификаторы. Поскольку биологические продукты демонстрируют естественные изменения формы, размера и цвета, система должна быть достаточно гибкой и надежной, чтобы справляться с этой изменчивостью. Комбинации морфологических, цветовых, текстурных признаков изображения могут быть использованы для разработки эффективных систем машинного зрения [35].

Морфологические особенности иллюстрируют внешнюю оболочку объекта. Современные алгоритмы могут извлекать морфологические признаки, описывающие базовые характеристики размера: площадь, периметр, ограничивающий прямоугольник, центр тя- жести, моменты - нормальные, центральные и инвариантные, длину, ширину и дескрипторы формы. Полученные данные также могут быть использованы для изучения влияния селекции и технологий на форму зерен.

Использование следующих морфологических параметров - длина зерна, ширина, максимальный диаметр, длина контура, площадь, соотношение сторон, нормированные центральные моменты, дескрипторы Фурье границы, в том числе радиальные - для классификации зерен было изучено в работе [36].

Цветовыми дескрипторами, которые можно извлечь из цветного изображения объекта, являются средние значения, дисперсии, диапазоны, моменты и гистограммы цветов RGB, a также средние значения, дисперсии, диапазоны тона (H), насыщенности (S) и интенсивности (I). В работе [36] приведены сравнительные результаты классификации на основе различных групп дескрипторов рис. 1, а также получен ранжированный список 20 наиболее эффективных цветовых дескрипторов.

Текстурные характеристики описывают распределение цвета в изображении относительно пространственных координат, т.е. позволяют учесть не только распределение яркостей, но и относительное расположение пикселей в изображении. То есть текстурные дескрипторы должны описывать такие свойства изображения, как размер зерна, плавность изменения цвета, хаотичность и нерегулярность [18]. Цифровые изображения двух различных объектов могут состоять из одного и того же количества пикселей и точно таких же гистограмм цвета, но, если распределение цвета неодинаково, они могут иметь совершенно отличный друг от друга вид. Тем не менее, эти два объекта, если они классифицируются с использованием простых цветовых признаков, будут распознаны как похожие объекты.

Известно много статистических и структурных подходов к математическим методам описания текстуры изображения: автокорреляционные функции, авторегрессионные модели, оптические преобразования, цифровые преобразования, структурные элементы и др. В настоящее время в задачах классифи- 
Распознавание изображений элементов зерновых смесей методами глубокого обучения ...

кации элементов зерновых смесей чаще всего используются матрица сочетаемости яркостей (GLCM) [37] и матрица длины кластера уровня яркости (GLRM) [38]. Матрица GLCM описывает распределении интенсивностей уровня серого как относительное положения пикселей с различной интенсивностью. Матрица GLRM описывает появление последовательных одинаковых по интенсивности пикселей, повторяющихся в определенном направлении. Для этих матриц вычисляются описывающие их дескрипторы, которые являются и текстурными дескрипторами. На основе матрицы GLCM - строчное среднее, строчная дисперсия, энергия, энтропия, контраст, корреляция, однородность, кластерный коэффициент, на основе матрицы GLRM - короткая серия, длинная серия, неоднородность, длина неоднородности, наполненность, энтропия [39].

Сравнительные результаты классификации на основе морфологических, цветовых и текстурных признаков с использованием полносвязной нейронной сети показаны на рис. 1.
В настоящее время в задачах компьютерного зрения широко используются нейронные сети глубокого обучения, показывающие впечатляющие результаты. В данной работе методом вычислительного эксперимента проведено исследование возможностей и особенностей применения таких нейронных сетей для решения задачи распознавания изображений элементов зерновых смесей на данных, предоставленных «Воронежсельмаш».

\section{МЕТОДИКА ЭКСПЕРИМЕНТА}

Одной из лучших библиотек, использующихся для глубокого обучения, является TensorFlow. Это библиотека с открытым исходным кодом для численного расчёта с использованием графа потока данных, разработанная Google. Узлы на графе представляют собой математические операции, в то время как ребра графа представляют собой многомерные массивы данных (тензоры), передаваемые между ними. По своему функционалу и возможностям TensorFlow заслуженно считается очень мощным инструментом для

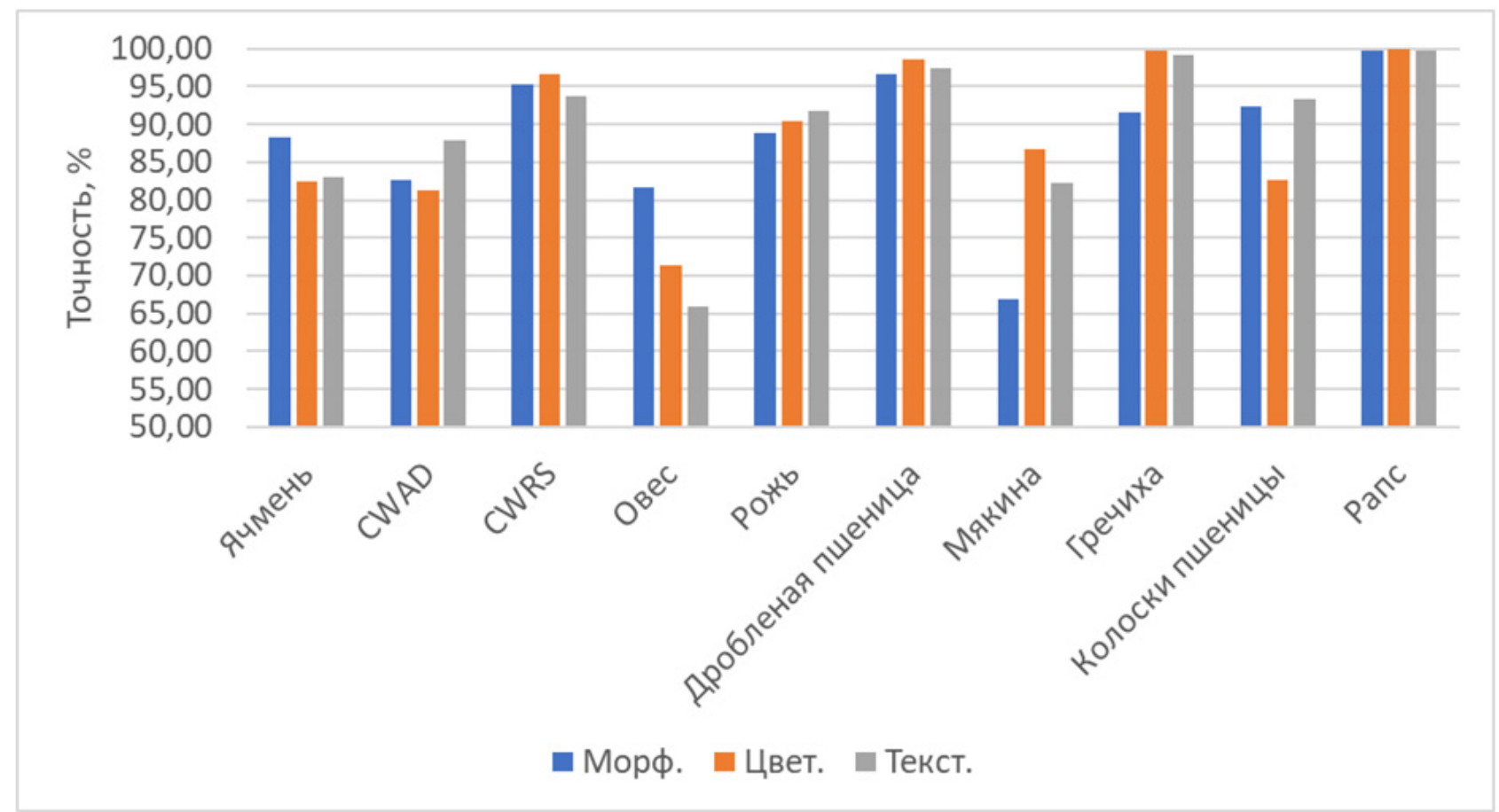

Рис. 1. Сравнение точности классификации зерен и элементов засоренности на основе морфологических, иветовых и текстурных признаков пяти видов зерна: ячмень, два сорта пшеницы (CWAD и CWRS), овес, рожь и пяти видов примесей: дробленая пшениць, мякина, гречиха, колоски пшеницы и рапс 
(a)
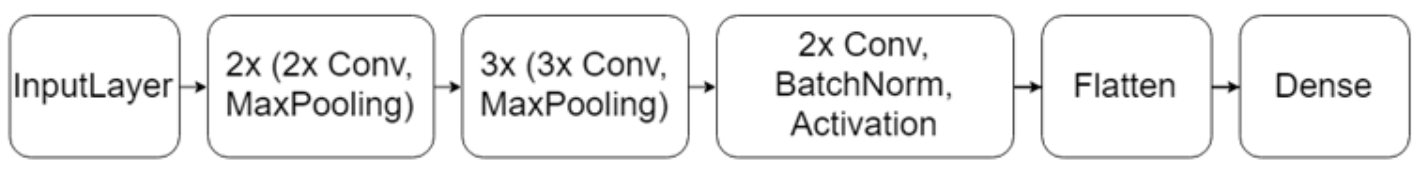

(б)
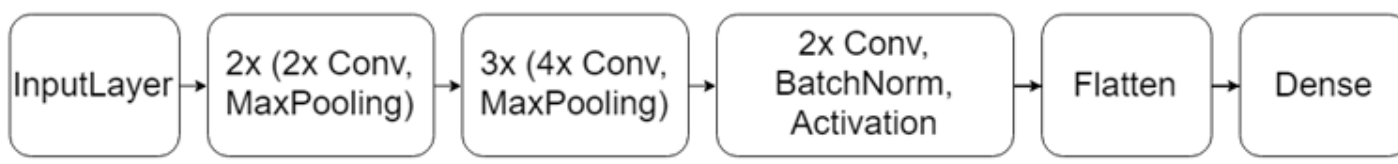

(B)
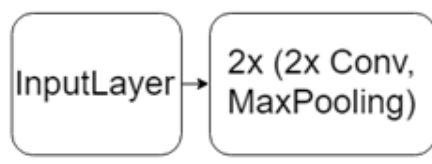

13x (Conv, BatchNorm, Activation, DepthConv,

BatchNorm, Activation)
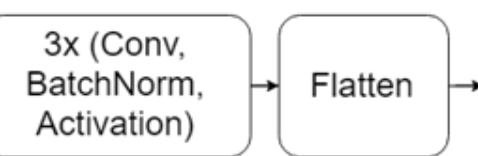

Dense

Puc. 2. Архитектура сетей: (а) VGG16, (б) VGG19, (в) MobileNet

разработки, однако является довольно сложной для использования библиотекой и требует длительного предварительного изучения своих особенностей и архитектуры. Чтобы облегчить её использование, была разработана библиотека Keras, являющаяся своего рода «оберткой» для TensorFlow.

Keras не занимается низкоуровневыми операциями, такими как работа с тензорами, свёртка и т. д. Вместо этого используется библиотека, которая выступает в качестве бекэнда: TensorFlow, Theano или CNTK. Тип модели в Keras называется последовательностью, которая представляет собой стек слоёв. После обучения можно использовать полученную модель для прогнозирования результатов на новых данных.

VGG16 - это нейронная сеть глубокого обучения, разработанная Visual Geometry Group для распознавания объектов на изображениях, состоящая из 16 слоёв [40]. На вход данная сеть получает RGB изображение и выдаёт на выходе в формате one hot encoding данные о том, к какому классу относится объект на изображении. Она содержит два основных блока. Первый выделяет характерные признаки в изображении и состоит из чередующихся каскадов свёртки и подвыборки. Сначала идут два каскада свёртка-свёртка-подвыборка, а затем три каскада свёртка-свёртка-свёртка-подвыборка. При подвыборке производится выбор Max Pooling (максимального значения). Другой блок выполняет задачу классифицирования объекта на изображении в соответствии с данными, полученными на предыдущем шаге. В пер-

вых двух полносвязных слоях находится 4096 нейронов, а в третьем - 1000 нейронов, что соответствует количеству классов объектов.

VGG19 - еще одна нейронная сеть другой, но близкой архитектуры от Visual Geometry Group, содержащая уже 19 слоёв, что можно видеть на рис. 2. Сеть MobileNet [41] имеет более отличающуюся структуру, что позволяет получать с помощью неё выходные файлы обученных сетей меньшего размера (приблизительно 50 МБ против 117 МБ и 174 МБ у VGG16 и VGG19 соответственно), которые проще использовать во встраиваемых системах и ПО для смартфонов.

В ходе исследования был разработан классификатор семян красного льна, зелёного льна, маша, нута, подсолнечника, пшеницы обычной, пшеницы красной, пшеницы стекловидной, свёклы, чечевицы, ячменя и кедра. Часть изображений, рассмотренных в исследовании, отображены на рис. 3. Представим данные о выборке в виде табл. 1. Ключевая сложность задачи классификации в данном случае заключается в том, что изображения имеют малый размер, который равняется в среднем $20 \times 10$ пикселей, а в наихудшем в выборке случае - $6 \times 6$ пикселей. Таким образом, исходные изображения несут в себе ограниченный объём информации, что усложняет задачу классификации.

Программный модуль был написан на языке программирования Python в среде разработки Jupyter Notebook с применением таких библиотек, как Tensorflow, Keras, CUDA, Pandas и ряда других. В качестве тестовых моделей сетей глубокого обучения использовались VGG16, VGG19 и MobileNet. 


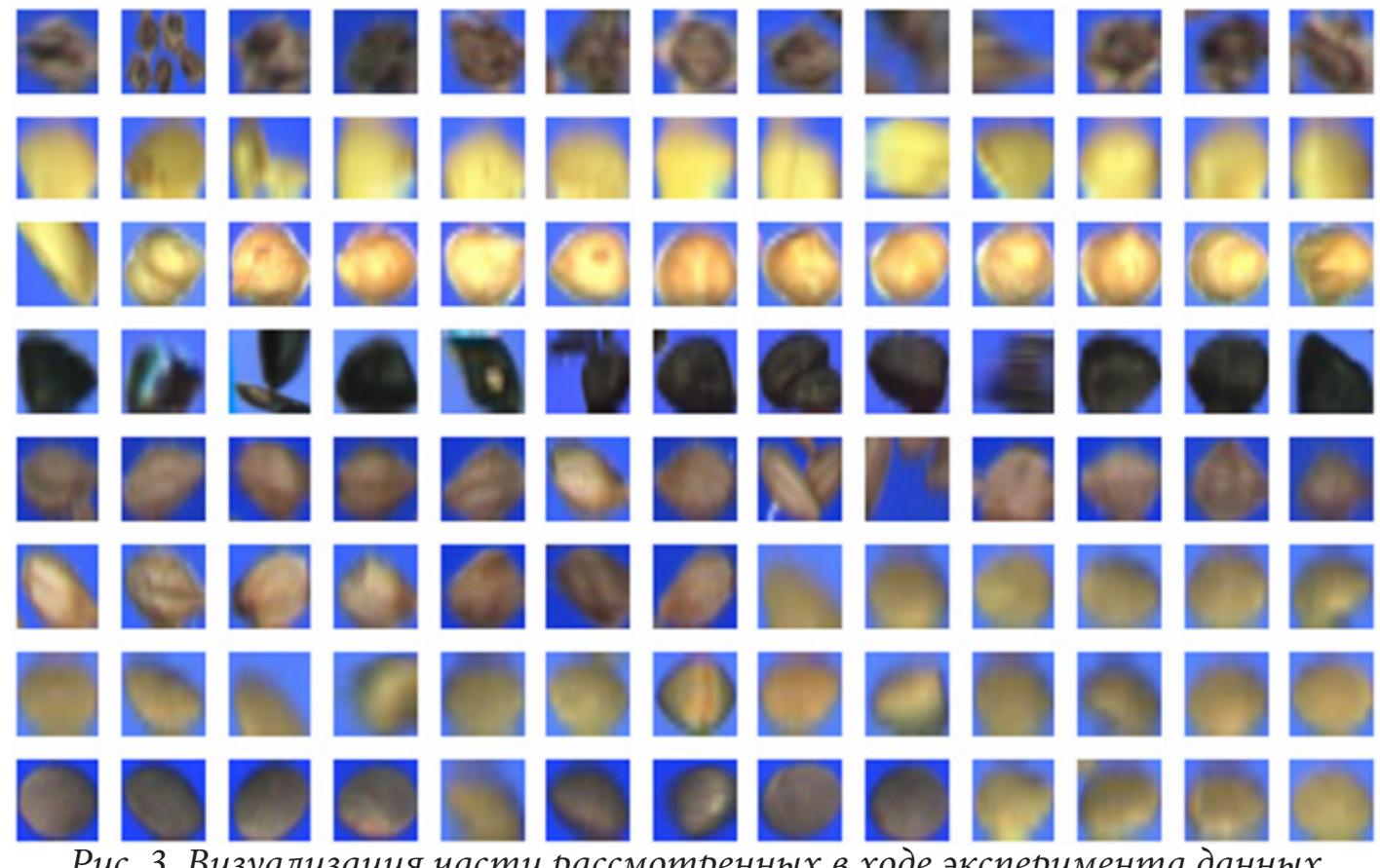

Рис. 3. Визуализация части рассмотренных в ходе эксперимента данных

Таблица 1

Описание исследованной выборки

\begin{tabular}{|l|l|l|l|}
\hline \multicolumn{1}{|c|}{ Название } & $\begin{array}{c}\text { Название } \\
\text { англоязычное }\end{array}$ & $\begin{array}{c}\text { В обучающей } \\
\text { выборке, шт }\end{array}$ & $\begin{array}{c}\text { В тестовой } \\
\text { выборке, шт }\end{array}$ \\
\hline Красный лён & Linum Red & 480 & 119 \\
\hline Зелёный лён & Linum Green & 270 & 52 \\
\hline Маш & Mung bean & 248 & 45 \\
\hline Нут & Cicer & 112 & 28 \\
\hline Подсолнечник & Helianthus & 95 & 23 \\
\hline Пшеница обычная & Wheat & 488 & 122 \\
\hline Пшеница красная & Wheat Red & 362 & 72 \\
\hline Пшеница стекловидная & Wheat Vitreous & 284 & 58 \\
\hline Свёкла & Beet seeds & 398 & 56 \\
\hline Чечевица & Lentil & 415 & 73 \\
\hline Ячмень & Hordeum & 213 & 42 \\
\hline Кедр & Cedrus & 131 & 32 \\
\hline
\end{tabular}

Вычислительные эксперименты выполнялись на компьютере с 12 ГБ оперативной памяти, процессором Intel Core i3-4030U и видеокартой NVIDIA GeForce 840M. Были установлены библиотеки Python 3.6.3, Anaconda 5.0.1, CUDA Toolkit 8.0, cuDNN v6.0, TensorFlow 1.4.0 и Keras версии 2.1.2. Обучение производилось в течение 20 эпох (epochs).

Основной особенностью сетей глубокого обучения является их способность самостоятельно выделять признаки на изображениях.
В связи с этим возникает вопрос об относительном вкладе морфологических, цветовых и текстурных особенностей изображения в точность распознавания при использовании сетей глубокого обучения. Это важно в том числе при решении задачи фотосепарации элементов зерновых смесей в потоке.

Для проведения экспериментов из исходных данных с помощью среды MATLAB было получено два набора модифицированных изображений. В первом наборе сохране- 


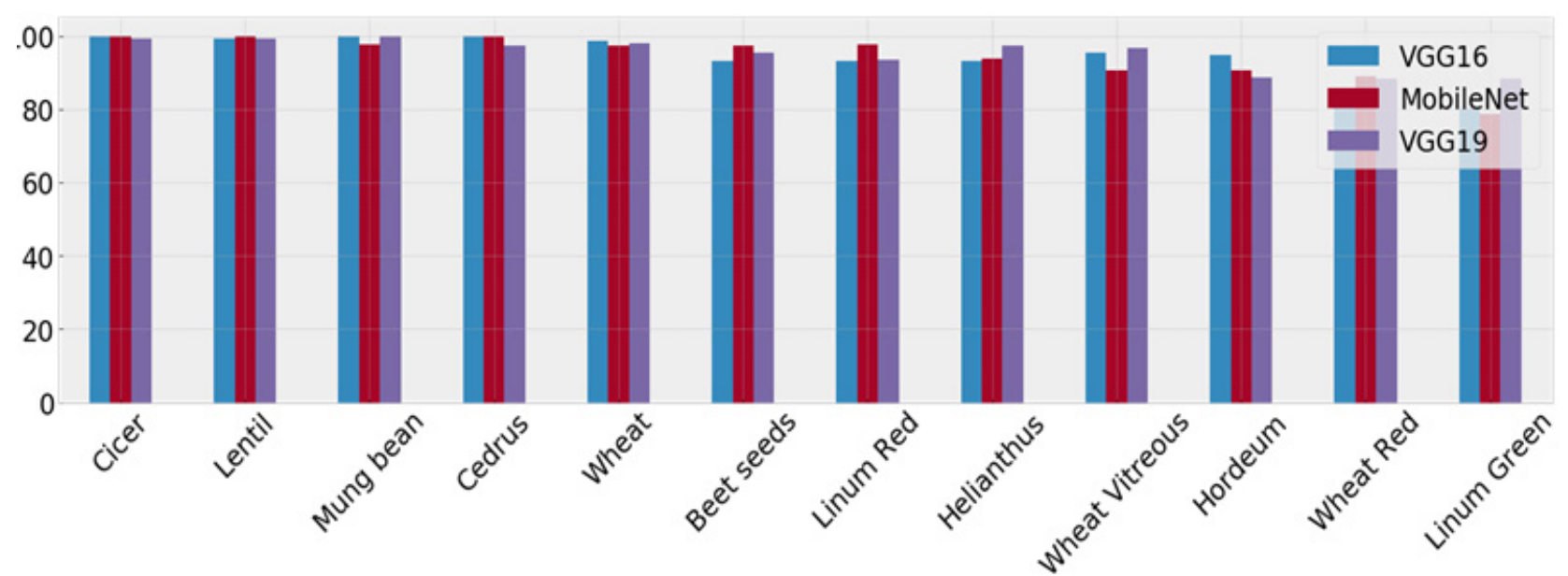

Рис. 4. Точность классификации зёрен в процентном соотночении для различных архитектур сетей

на только информация о контурах исходных изображений, а во втором - только о текстуре и цвете. Контуры были получены с помощью стандартной функции imcontour из пакета Image Processing Toolbox, разрешение модифицированных изображений, содержащих только контур зерна, совпадает с разрешением исходных изображений.

Как видно из изображений, представленных на рис. 3, фон каждого из них является синим. Чтобы убрать его из рассмотрения и получить именно интересующую текстуру, были использованы особенности цветовой модели RGB, в которой представлены исходные данные. Таким образом, для выделения текстуры были убраны участки, на которых значения синего канала $B \in[100,255]$ (т. е. части изображения заведомо синего оттенка, притом, что рассматриваемые зёрна синими не являются) и с помощью функции imcrop была получен квадратный фрагмент оставшихся данных, сохранив таким образом цветовые и текстурные особенности изображений элементов зерновых смесей. Были получены такие объекты размерами $3 \times 3,5 \times 5$, $7 \times 7,9 \times 9$ и $11 \times 11$ пикселей, составившие второй набор модифицированных изображений.

\section{ВЫЧИСЛИТЕЛЬНЫЕ ЭКСПЕРИМЕНТЫ И ИХ ОБСУЖДЕНИЕ}

При использовании TensorFlow c GPU, базирующимся на технологии CUDA, на обучение в среднем для разных моделей было за- трачено 41,9833 минут. В случае выполнения обучения без использования CUDA, в режиме CPU, данный процесс обучения занимает около 13 часов. Поэтому дальнейшие вычисления производились только в режиме GPU. Всего 3146 экземпляров объектов использовалось для обучения и 350 для валидации, при обучении на обработку каждого изображения в среднем для разных архитектур сети было затрачено 0,8009 секунд. На выполнение классификации в среднем было затрачено 25,6669 секунд для 722 изображений, в том числе на каждое отдельное изображение 0,0355 секунды машинного времени. В ходе исследований точность классификации, усредненная по различным архитектурам сети и классам объектов, составила 94,6352 \%.

В частности, для VGG19 была достигнута средняя точность 95,0692 \%, для VGG16 94,1274 \% и для MobileNet - 94,7091 \%. При этом MobileNet имеет наилучшие показатели по общему времени обучения и времени классификации (1808,2264 секунд и 13,0165 секунд соответственно), против VGG16 $(2509,5991$ и 34,9570 секунд) и VGG19 (3241,6330 и 29,0271 секунд соответственно). Количество верно распознанных в ходе исследования изображений для различных нейросетей глубокого обучения в процентном соотношении представлено в табл. 2 и на рис. 4.

Вычислительные эксперименты на искусственных объектах (рис. 5), сохранивших только морфологические или цветовые и текстурные признаки, показали следующее. Для 
Распознавание изображений элементов зерновых смесей методами глубокого обучения ...

Таблица 2

Точность классификации семян для рассматриваемых нейросетей

\begin{tabular}{|l|l|l|l|}
\hline \multicolumn{1}{|c|}{ Название } & $\begin{array}{c}\text { Верно распознанных } \\
\text { VGG16, \% }\end{array}$ & $\begin{array}{c}\text { Верно распознанных } \\
\text { MobileNet, \% }\end{array}$ & $\begin{array}{c}\text { Верно распознанных } \\
\text { VGG19, \% }\end{array}$ \\
\hline Beet seeds & 93.2142 & 97.5000 & 95.3571 \\
\hline Lentil & 99.1780 & 100.0000 & 99.4520 \\
\hline Cedrus & 100.0000 & 100.0000 & 97.5000 \\
\hline Cicer & 100.0000 & 100.0000 & 99.2857 \\
\hline Helianthus & 93.0434 & 93.9130 & 97.3913 \\
\hline Hordeum & 94.7619 & 90.4761 & 88.5714 \\
\hline Linum Green & 80.3846 & 78.8461 & 88.4615 \\
\hline Linum Red & 93.2773 & 97.6470 & 93.6134 \\
\hline Mung bean & 100.0000 & 97.7777 & 100.0000 \\
\hline Wheat & 98.5245 & 97.3770 & 98.0327 \\
\hline Wheat Red & 83.8888 & 88.8888 & 88.3333 \\
\hline Wheat Vitreous & 95.5172 & 90.6896 & 96.8965 \\
\hline
\end{tabular}

объектов, несущих только морфологическими характеристики изображений элементов зерновых смесей, средняя точность классификации для всех рассмотренных архитектур нейросетей составляет 60,2585 \%, а для объектов, несущих только цветовые и текстурные признаки (с разрешением $9 \times 9$ пикселей) $90,1846 \%$. (a)
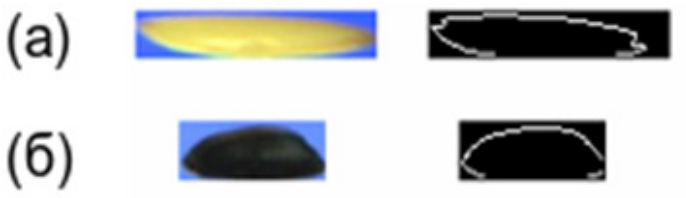

(B)

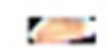

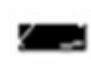

Рис. 5. Визуализачия исходного изображения, его контура и текстуры соответственно: (а) зерно кедра, (б) зерно подсолнечника, (в) зерно красной пшениць

Выбор разрешения объекта $9 \times 9$ пикселей для отражения цветовых и текстурных характеристик можно считать оптимальным, так как на подавляющем большинстве рассмотренных фотографий зёрен возможно найти участок текстуры данного размера.

При решении задачи классификации элементов зерновых смесей в потоке в фотосепараторе с линейными камерами возникает необходимость обрабатывать фрагменты изображений зерен размерами несколько пиксе- лей. Для исследования возможности использования для этих целей программного обеспечения на основе нейронных сетей глубокого обучения, были выполнены вычислительные эксперименты с искусственными объектами размерами $3 \times 3,5 \times 5$ и $7 \times 7$ пикселей, несущими только цветовые и текстурные характеристики реальных зерен. Средняя точность классификации для объектов размером $3 \times 3$ пикселя составила около $74 \%$, для объектов $5 \times 5-79 \%$, а для $7 \times 7-86 \%$, что представлено на рис. 6. Как видно, точность распознавания увеличивается с ростом размера изображения и, соответственно, объема содержащейся в изображении информации. Объекты размером $11 \times 11$ пикселя не рассматривались, так как уже для заметного процента имеющихся изображений зерен невозможно было выделить соответствующий фрагмент текстуры.

По результатам проведенных вычислительных экспериментов можно сделать следующие выводы. Программное обеспечение на основе нейронных сетей глубокого обучения может эффективно использоваться для анализа изображений элементов зерновых смесей. В данной работе для исследований были взяты три наиболее показательные архитектуры нейросетей, причем их модификация в целях подстройки под задачу распознавания 


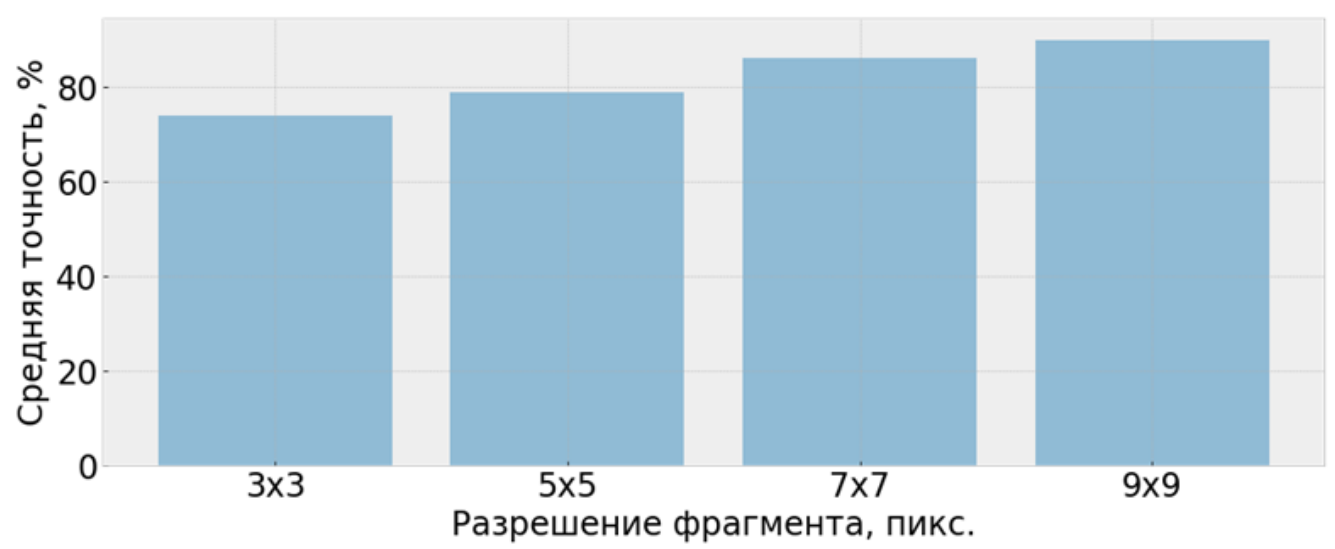

Рис. 6. Средняя точность классификации при различном разрешении фрагмента текстуры

изображений зерен не проводилась. Для таких архитектур существенно больший вклад в решение задачи распознавания вносят цветовые и текстурные признаки по сравнению с морфологическими. Особый интерес представляют полученные результаты распознавания искусственных объектов, содержащих фрагмент изображения зерна с сохранением только цветовых и текстурных признаков. Можно сделать вывод о перспективности разработки алгоритмов и программного обеспечения на основе глубокого обучения для потокового распознавания зерен в фотосепараторах.

По итогам большого количества выполненных вычислительных экспериментов наиболее эффективной оказалась нейросеть MobileNet. Она требует меньше времени на обучение, меньше времени на классификацию (что более важно) и даже сам обученный файл требует в несколько раз меньшее количество места в файловой системе, чем у конкурентов. Таким образом, MobileNet удобнее для использования во встраиваемых системах. Конечно, VGG19 показал несколько большую точность классификации, но это различие не столь велико (95.0692 \% против $94.7091 \%)$ и, при учёте других преимуществ, MobileNet представляется более перспективной для использования.

\section{ЗАКЛЮЧЕНИЕ}

Использование нейросетей глубокого обучения в реальных задачах классификации изображений элементов зерновых смесей требует разработки архитектуры сети, учитывающей специфику задачи. В частности, необходимо значительно увеличить точность распознавания по морфологическим характеристикам как минимум до результатов, показываемых полносвязными нейросетями с использованием вычисляемых дескрипторов. Также необходимо увеличение точности классификации только по цветовым и морфологическим признакам небольших фрагментов изображений. Решение этих проблем позволит, в достаточно близкой перспективе, разработать полностью самообучаемое программное обеспечения для фотосепараторов.

Результаты работы получены в рамках выполнения государственного задания Минобрнауки России по проекту № 8.3844.2017/4.6 «Разработка средств экспресс-анализа и классификации элементов неоднородного потока зерновых смесей с патологиями на основе интеграции методов спектрального анализа и машинного обучения».

\section{СПИСОК ЛИТЕРАТУРЫ}

1. Shaw, W. E. Machine vision for detecting defects on fruit and vegetables / W. E. Shaw // In Food Processing Automation I - Proceedings of the 1990 conference 50-59. St. Joseph, MI: ASAE. -1990. - P.50-59.

2. Tillet, $R$. D. Image analysis for agricultural processes: A review of potential opportunities / R. D. Tillet // Journal of Agricultural Engineering Research. - 1991. - № 50. - P. 247-258.

3. Barker, D. A. The use of ray parameters for the discrimination of Australian wheat varieties / 
Распознавание изображений элементов зерновых смесей методами глубокого обучения ...

Barker D. A., Vouri T. A., Hegedus M. R. and Myers D. G. // Plant Varieties and Seeds. - 1992. - № 5(1). - P. 35-45.

4. Barker, D. A. The use of slice and aspect ratio parameters for the discrimination of Australian wheat varieties / D. A. Barker, T. A. Vouri, and D.G. Myers // Plant Varieties and Seeds. - 1992. № 5(l). - P. 47-52.

5. Barker, D. A. The use of Fourier descriptors for the discrimination of Australian wheat varieties / D. A. Barker, T. A. Vouri and D. G. Myers // Plant Varieties and Seeds. - 1992.- № 5(2). P. 93-102.

6. Barker, D. A. The use of Chebychev coefficients for the discrimination of Australian wheat varieties / D. A. Barker, T. A. Vouri, M. R. Hegedus and D.G. Myers // Plant Varieties and Seeds. 1992. - № 5(2). - P. 103-111.

7. Crowe, T. G. Color line-scan imaging of cereal grain kernels / T. G. Crowe, X. Y. Luo, D. S. Jayas and N. R. Bulley // Applied Engineering in Agriculture. - 1997. - № 13(5). - P. 689694.

8. Draper, S. R. Preliminary observations with a computer based system for analysis of the shape of seeds and vegetative structures / S. R. Draper and A. J. Travis // Journal of the National Institute of Agricultural Botany. - 1984. - №16(3). P. 387-395.

9. Keefe, P. D. An automated machine vision system for the morphometry of new cultivars and plant genebank accessions / P. D. Keefe and S. R. Draper // Plant Varieties and Seeds 1(1): $1-11$.

10. Keefe, $P$. D. The measurement of new characters for cultivar identification in wheat using machine vision / P. D. Keefe and S. R. Draper // Seed Science and Technology. - 1986. - №14(3). P. 715-724.

11. Lai, F. S. Application of pattern recognition techniques in the analysis of cereal grains / F. S. Lai, I. Zayas, and Y. Pomeranz // Cereal chemistiy. 1986. - №63(2). - P. 168-172.

12. Majumdar, S. Textural features for automated grain identification / S. Majumdar, D. S. Jayas, S. J. Symons and N.R. Bulley // CSAE Paper No. 96-602. Saskatoon, SK: CSAE, 1996.

13. Majumdar, S. Classification of various grains using optical properties / S. Majumdar,
D. S. Jayas, J. L. Hehn and N. R. Bulley // Canadian Agricultural Engineering. - 1996. - №.38(2). P. 139-144.

14. Majumdar, S. Single-kernel mass determination for grain inspection using machine vision / S. Majumdar and D. S. Jayas // Applied Engineering in Agriculture. - 1999. - №15(4). P. 357-362.

15. Majumdar, S. Classification of bulk samples of cereal grains using machine vision / S. Majumdar and D. S. Jayas. // Journal of Agricultural Engineering Research. - 1999. - №73(1). - P. 35-47.

16. Majumdar, S. Classification of cereal grains using machine vision. I. Morphology models / S. Majumdar and D. S. Jayas // Transactions of the ASAE. - 2000. - № 43(6). - P. 1669-1675.

17. Majumdar, S. Classification of cereal grains using machine vision. II. Color models / S. Majumdar and D. S. Jayas // Transactions of the ASAE. - 2000. - №43(6). - P.1677-1680.

18. Majumdar, S. Classification of cereal grains using machine vision. III. Texture models / S. Majumdar and D. S. Jayas // Transactions of the ASAE. - 2000. - №43(6). - P.1681-1687.

19. Majumdar, S. Classification of cereal grains using machine vision. IV. Combined morphology, color, and texture models / S. Majumdar and D. S. Jayas. // Transactions of the ASAE. 1988. - № 43(6). - P. 1689-1694.

20. Myers, D. G. The application of image processing techniques to the identification of Australian wheat varieties / D. G. Myers and K. J. Edsall // Plant Varieties and Seeds. - 1989. № 2(2). - P. 109-116.

21. Neuman, M. Discrimination of wheat class and variety by digital image analysis of whole grain samples / M. Neuman, H. D. Sapirstein, E. Shwedyk and W. Bushuk // Journal of Cereal Science. - 1987. - № 6(2). - P. 125-132.

22. Paliwal J. Grain kernel identification using kernel signature / J. Paliwal, N. S. Shashidhar and D. S. Jayas // Transactions of the ASAE. - 1999. №42(6). - P.1921-1924.

23. Sapirstein, H. D. Quantitative determination of foreign material and vitreosity in wheat by digital image analysis / H. D. Sapirstein and W. Bushuk // lnICC'89 Symposium: Wheat EndUse Properties. H. Salovaara (ed.). Lahiti, Finland, 1989. 


\section{А. А. Крыловеикий, Д. М. Суходолов}

24. Sapirstein, H. D. An instmmental system for cereal grain classification using digital image analysis / H. D. Sapirstein, M. Neuman, E. H. Wright, E. Shwedyk and Bushuk W. // Journal of Cereal Science. - 1987. - № 6(1). - P. 3-14.

25. Symons, S. J. Relationship between oat kernel weight and milling yield / S. J. Symons and R. G. Fulcher // Journal of Cereal Science. 1988. - №7(3). - P. 215-217.

26. Symons, S. J. 1988b. Determination of variation in oat kernel morphology by digital image analysis / S. J. Symons and R. G. Fulcher // Journal of Cereal Science. - 1988. - № 7(3). - P. 219-228.

27. Zayas, I. Discrimination between wheat classes and varieties by image analysis / I. Zayas, F. S. Lai and Y. Pomeranz // Cereal Chemistry. 1986. - №63(1). - P. 52-56.

28. Zayas, I. Discrimination of whole from broken com kernels with image analysis / I. Zayas, H. Converse and J. Steele // Transactions of the ASAE. - 1990. - №35(5). - P. 1642-1646.

29. Zayas, I. Discrimination between Arthur and Arc an wheats by image analysis / I. Zayas, Y. Pomeranz and F. S. Lai // Cereal Chemistry. 1985. - № 62(6). - P. 478-480.

30. Zayas, I. Discrimination of wheat and non-wheat components in grain samples by digital image analysis / I. Zayas, Y. Pomeranz and F. S. Lai // Cereal Chemistry. - 1989. - №66(3). P. 233-237.

31. Wigger, W. D. Classification of fungal-damaged soybeans using color-image processing / W. D. Wigger, M. R. Paulsen, J. B. Litchfield and J. B. Sinclair // ASAE Paper No. 883053. - 1988. - St. Joseph, MI: ASAE.

32. Karunakaran, C. Machine Vision Systems for Agricultural Products / C. Karunakaran, N. S. Visen, J. Paliwal, G. Zhang, D. S. Jayas,

Крыловецкий А. А. - канд. физ.-мат. наук, доцент кафедры цифровых технологий, факультет компьютерных наук, Воронежский государственный университет.

E-mail: aakryl@cs.vsu.ru

Суходолов Д. М. - магистрант кафедры цифровых технологий, факультет компьютерных наук, Воронежский государственный университет. E-mail: sukhodolov.denis@gmail.com
N. D. G. White // CSAE Paper No. 01-305. 2001. - Mansonville QC: CSAE/SCGR.

33. Paliwal, J. Digital image analysis of grain samples for potential use in grain cleaning (Ph. D. thesis) / J. Paliwal; Department of Biosystems Engineering, University of Manitoba, Winnipeg, MB, Canada, 2002. - 247 p.

34. Visen, N. S. Machine Vision Based Grain Handling System (Ph.D. thesis) / N. S. Visen; Department of Biosystems Engineering, University of Manitoba, Winnipeg, MB, Canada. - 175 p.

35. Computer Vision Technology for Food Quality Evaluation / Edited by Da-Wen Sun; Academic Press, Elsevier. - 2016. - 635 P.

36. Paliwal, J. Cereal grain and dockage identification using machine vision / J. Paliwal, N. S. Visen, D. S. Jayas, N. D. G. White // Biosystems Engineering. - 2003. - №85(1). - P. 51-57.

37. Haralick, R. M. Texture features for image classification. IEEE Transactions on Systems / R. M. Haralick, K. Shanmugam and I. Dinstein // Man and Cybernetics. - 1973. - № 3(6). - P. 610621.

38. Galloway, M. M. Texture analysis using gray level run length // Galloway M.M. // Computer graphics and image processing. - 1975. № 4. - P. 172-179.

39. Гонсалес, Р. Цифровая обработка изображений / Р. Гонсалес, Р. Вудс. - М. : Техносфера, 2012. - 1104 c.

40. Simonyan, K. Very deep convolutional networks for large-scale image recognition / K. Simonyan, A. Zisserman // CoRR. - 2014. № 1409.1556. - 14 c.

41. Howard, A. G. MobileNets: Efficient Convolutional Neural Networks for Mobile Vision Applications / A. G. Howard [и др.] // CoRR. 2017. - № 1704.04861. - 9 c.

Krylovetsky A. A. - Ph. D. in Physics and Mathematics, Associate Professor of Department of Digital Technologies, Voronezh State University. E-mail: aakryl@cs.vsu.ru

Sukhodolov D. M. - Graduate student of the Department of digital technology, Voronezh State University.

E-mail: sukhodolov.denis@gmail.com 